\title{
Opioids can be useful in the treatment of headache
}

\author{
Cinzia Finocchi $\cdot$ Erica Viani
}

(C) Springer-Verlag Italia 2013

\begin{abstract}
The use of opioids in headache treatment is very controversial. In the migraine acute attack use of short-acting opioids is not recommended by the principal guidelines but is frequent in North American emergency departments. Their efficacy in migraine acute attack has not been extensively studied but seems to be similar to nonsteroidal anti-inflammatory drugs and metoclopramide. Opioids have been never compared to triptans. The principal concerns about the use of opioids regard the possible association with an increased risk of medication-overuse headache and chronic migraine and the risk of abuse and dependence. These risks have to be considered but not overestimated. The association between frequent use and increased risk of chronic migraine has been observed for almost all categories of acute migraine attack drugs. Compared to the reference category of acetaminophen, risk of chronic migraine for opioid use is only moderately higher (with an $\mathrm{OR}=1.48$ ). In some cases, when treatment with triptans, non-steroidal anti-inflammatory drugs, or ergotamines is contraindicated or simply ineffective, a judicious prescription of a short-acting opioid for severe migraine attacks can be considered. Chronic migraine is a highly disabling condition. Although the options for prophylaxis therapy of migraine have expanded and improved considerably over recent years, chronic migraine remains very difficult to treat. The results coming from small clinical series are described, suggesting that in expert hands daily long-acting opioids provide an option for the
\end{abstract}

C. Finocchi $(\bowtie) \cdot$ E. Viani

Dipartimento di Neuroscienze, Riabilitazione, Oftalmologia,

Genetica e Scienze Materno-Infantili, University of Genova,

Largo Daneo 3, 16132 Genova, Italy

e-mail: cfinocchi@neurologia.unige.it treatment of some individuals with chronic intractable headaches.

Keywords Opioids $\cdot$ Migraine $\cdot$ Headache $\cdot$ Preventive therapy $\cdot$ Acute treatment $\cdot$ Chronic migraine

\section{Introduction}

Migraine is a common and potentially disabling disorder with a wide range of available effective pharmacologic and non-pharmacologic (i.e. behavioural) therapies [1-4]. The two principal aims of migraine therapy are to rapidly and consistently relieve pain, minimize recurrence and restore patient's ability to function in acute attack and to reduce the frequency and intensity of attacks with appropriate preventive therapies when the frequency of migraine attacks is elevated. The drugs utilized in acute attack therapy include nonspecific agents used for headache and other pain disorders [e.g. acetaminophen, aspirin, non-steroidal antiinflammatory drugs (NSAIDs), and opioids], and migrainespecific agents (e.g. triptans). Both the EFNS Guidelines [2] and the US Headache Consortium Guidelines [4] suggest an individualized stratified-care approach in which the choice of acute agent(s) must change taking into account migraine attack frequency and severity, degree of disability, associated headache symptoms, previous response to medications and presence of other disorders. The US Headache Consortium Guidelines consider that opioids are second- or thirdtier choices for migraine following simple analgesics and migraine-specific medications, while European Guidelines do not suggest their use at all.

Migraine is usually considered a chronic disorder with a benign course, but it is becoming clear that a subgroup of patients worsens over time. During this progression the 
frequency of headaches increases with evolution from an episodic to a chronic migraine (CM) [5]. Most patients with CM overuse symptomatic medications and may also be classified in Group 8 of the second edition of the international classification of headache disorders (ICHD-2R) ("Headache attributed to a substance or its withdrawal") as medication-overuse headache $(\mathrm{MOH})$ [6].

The principal concern about the use of opioid in migraine acute attack regards the possible association with an increased risk of $\mathrm{MOH}$ and $\mathrm{CM}$ [7, 8]. In addition, opioids may be misused or abused, leading to opioid abuse or dependence [9]. Despite these concerns and despite the recommendations of the principal guidelines that physicians should limit use of all narcotic analgesics in the treatment of migraine, opioids are frequently used, especially in the emergency department (ED) setting [10].

Taking into account that our arsenal of evidence-based, effective and specific therapies for acute migraine headache of moderate to severe intensity is limited, does a role exist for short-acting opioids in the migraine acute attack?

$\mathrm{CM}$ is a highly disabling condition, represents a great proportion of patients presenting to headache centres and accounts for a disproportionate share of the direct and indirect costs attributable to migraine diagnosis and treatment [11]. While the options for prophylaxis therapy of migraine have expanded and improved considerably over recent years, $\mathrm{CM}$ remains very difficult to treat. The medical literature contains relatively few reports of randomized controlled trials about treatment of CM [12-14] and relatively few patients receive a benefit from the proposed therapies. A further hope for patients with CM might come from the neurostimulation techniques [15]. All practicing clinicians know that a large subpopulation of patients with a treatment-refractory $\mathrm{CM}$ remains. Therefore, some data suggest that patients with CM may become significantly less likely to respond to therapeutic intervention if they have been experiencing daily headache for 6 months or more $[16,17]$.

Taking into account this scenery, can we disregard the results obtained in chronic headache patients by long-acting opioid therapies even if they come from small clinical series and not from controlled trials [18-21]?

\section{Role of short-acting opioids in the migraine acute attack}

In the US population, over $18.7 \%$ of migraineurs routinely uses triptans. Among the nonspecific prescribed medications, opioids are used by $11.1 \%$. Another $6 \%$ uses barbiturate-containing medications (often combined with codeine) [22]. Parenteral opioids are very frequently used in the ED setting: in $51 \%$ of all migraine visits to US EDs and in $59.6 \%$ of patients with migraine acute attack in.
Canadian EDs, as first-line treatment [23, 24]. Meperidine, the individual opioid agent used most frequently, is administered in $36 \%$ of all US migraine visits [23]. There is a wide variation in the pharmacological treatment of migraine, variability that is especially evident with the use of opioids. Vinson [23] find that, in several different acute care settings, the use of opioid medications for migraine headaches varied from 16 to $71 \%$. It is evident that acute migraine management in these EDs does not meet current consensus guidelines. Some factors can influence the physicians' behaviour: patients who have taken anti-headache medications prior to ED presentation are two and half times more likely to receive narcotics and patients treated with first-line narcotics are also more than twice as likely to have repeated ED evaluations for headache [24]. It seems reasonable that the patients, who physicians judge good candidates for narcotic therapy in the ED, represent a subpopulation particularly difficult to treat, even if we cannot exclude that some of these patients may well represent a population who are particularly demanding of narcotics. It is interesting to note that patients treated with opioids were discharged from the ED much more quickly than those treated with non-narcotic agents.

In Europe the use of opioid is less common. In a French study [25] non-opioid analgesics excluding NSAIDs, and NSAIDs, were most commonly prescribed as acute treatment; opioids were not used at all. It should be noted, however, that it took more than $48 \mathrm{~h}$ for symptom resolution in $36 \%$ of 92 follow-up patients.

Both in Europe and in US parenteral triptans, that are expected to have a superior efficacy on a basis of placebocontrolled trials, are rarely used, probably because of the fear of cardiovascular adverse events [26, 27].

The efficacy of opioids in migraine acute attack management is not clearly established.

Meperidine is the opioid agent most frequently used in ED. A recent meta-analysis [28] evaluates all randomized controlled trials, to determine the relative efficacy and adverse effect profile of meperidine compared with nonopioid active comparators for the treatment of acute migraine. 19 clinical trials have been identified: four trials involving 254 patients compare meperidine to dihydroergotamine (DHE), four trials involving 248 patients compare meperidine to an antiemetic, and three trials involving 123 patients compare meperidine to ketorolac. Meperidine is less effective than DHE e.v., but is not less effective than antiemetics and ketorolac. The drug is rather well tolerated.

The review of agency for healthcare research and quality [29] on acute migraine treatment in emergency settings concludes that opioids are demonstrated more effective than placebo for pain relief with a moderate strength of evidence. The trials that compare opioids with hydroxyzine, other opioids (i.e., nalbuphine, meperidine), 
methotrimeprazine, metoclopramide, neuroleptic agents, NSAIDs, and DHE have insufficient strength of evidence. The mixed treatment analysis demonstrates with low strength of evidence that opioids are similarly effective to NSAIDs and metoclopramide. There is insufficient strength of evidence for headache recurrence when comparing opioids and other active agents. No trial compares opioids and triptans in the acute migraine attack.

Tramadol hydrogen chloride is an atypical opioid that weakly binds the mu opioid receptor and also inhibits serotonin and norepinephrine re-uptake. Tramadol is better tolerated than other opioids because of its low impact on the respiratory, cardiac, and gastrointestinal systems at therapeutic doses, but its efficacy in migraine acute attack is not proven $[30,31]$.

Overuse of short-acting opioids may be associated to worsening of migraine, increasing the risk of $\mathrm{CM}$ and $\mathrm{MOH}$. Clinical-based and population-based longitudinal studies support an association between CM and opioid use [32]. This is also true for patients suffering from episodic migraine assuming daily opioids (but also other analgesics) for other pain disorders, while in subjects without migraine, excessive opioid use does not seem to induce headaches [32]. In the American Migraine Prevalence and Prevention (AMPP) study [33], compared to the reference category of acetaminophen use, risk of transformed migraine (TM)/chronic migraine is higher for opioid use (with an $\mathrm{OR}=1.48$, moderately but not highly elevated), and also increased for barbiturate use $(\mathrm{OR}=1.73)$. Critical level of exposure is around 8 days per month, and the effect is more evident in men $(\mathrm{OR}=2.76)$ than in women $(\mathrm{OR}=1.28)$.

The association between frequent use and increased risk of TM/CM has been observed for almost all categories of acute migraine attack drugs. The relationship is complex: emerging evidence suggests that the choice of acute treatment and the frequency of use have a major influence and that the majority of acute medications, opioids, barbiturate-containing combination analgesics, as well as triptans and ergots, potentiate this risk of progression [34]. The frequent use of triptans, that are considered first-choice drugs for the moderate/severe migraine acute attack, has been shown to result in migraine progression with fewer doses compared with ergots and analgesics and with a shorter interval between first intake and daily headache (1.7 years for triptans, 2.7 years for ergots, and 4.8 years for common analgesics) [35]. In the clinical practice, therefore, rarely patients use only one type of acute attack medication. In the AMPP study [33] only $6.2 \%$ reports using opioids exclusively, $93.8 \%$ uses more than one class of acute headache medication including NSAIDs, triptans, ergots and barbiturates among others. The prevention of progression and worsening of migraine includes the control and elimination of all the identified risk factors without overestimate the role of a single factor (opioids agents, for example). Therefore, to say that a factor is associated to a condition does not mean that the factor causes the condition: it is possible that frequent use of opioids (and other drugs) for migraine acute attacks is simply a marker of a serious disorder which is evolving to the chronification. The observation that in many patients with $\mathrm{CM}$ and $\mathrm{MOH}$ withdrawn from acute medication before initiating preventive drug therapy might not be necessary is an indirect confirmation of the last hypothesis [36].

The pathophysiology of CM is very complex: structural or morphologic changes have been described in many brain structures such as reduced cortical gray matter of the pain processing areas of the brain and iron accumulation in the periaqueductal gray matter, red nucleus, and basal ganglia structures [37, 38]. Of special interest are the reversible hypometabolism in the insula, thalamus, anterior cingulate, and parietal lobe and the sustained hypometabolism in the orbitofrontal cortex observed in MOH. All these modifications may have a role in transformation to $\mathrm{CM}$ or may be physiologic responses to repeated activation of nuclei involved in central pain processing [39]. A central sensitivation seems to be the common result of the alterations observed in $\mathrm{CM}$ and opioids may contribute to this process by means of a paradoxical increase in pain sensitivity in response to opioids, which is known as opioid-induced hyperalgesia [40]. This phenomenon may be mediated both through an activation of the central glutamatergic system and through a regulation of neuronal function by means of glia [40].

Prophylactic drugs may modulate sensitivation and hyperalgesia [41]. Amitriptyline, propranolol, verapamil, lamotrigine, gabapentin, magnesium, melatonin delay the development of tolerance to opioids and increase their analgesic effects. The risk of $\mathrm{CM}$ and $\mathrm{MOH}$ in a patient in prophylactic therapy who assumes opioids as attack therapy may be consistently lower.

The last risk we have to consider about the use of shortacting opioids by migraine patients is the risk of narcotic abuse. In a study [42] $7 \%$ of patients discharged with a diagnosis of migraine used injections of morphinomimetic drugs once a week or more and were regarded as drug abusers by their physicians. Therefore, the $19 \%$ of a series of patients dependent from opioids had headache as a reason for initiation of use [43].

Taking into account all the concerns we mentioned, we can conclude that the role of short-acting opioids in migraine acute attack is limited but not absent. In some cases, when treatment with triptans, NSAIDs, or ergotamines is contraindicated or simply ineffective, a judicious prescription of a short-acting opioid as "rescue" from severe migraine would appear both sensible and medically 
appropriate. Opioids should be used 2 days a week maximum, to avoid the risk of chronification and $\mathrm{MOH}$. In all patients using short-acting opioids for refractory migraine acute attacks, a preventive therapy could be indicated even in presence of an attack frequency not particularly elevated.

\section{Role of long-acting opioids in chronic migraine}

Migraine is usually considered a benign disorder, but a subgroup of patients worsens over time. A migraine that worsens progressively until it becomes daily or almost daily falls back into the field of so-called chronic daily headache, and does not have a universally recognized place within the current international headache classification system. In ICHD-2R this headache type is included in CM that is characterized by the presence of at least 15 days of headache per month for at least three consecutive months, with headache having the same clinical features of migraine without aura for at least eight of those 15 days [6]. Patients whose migraine can be defined CM are very heterogeneous and those who really have a daily headache have few chances to benefit from the current therapies and should be distinguished in a specific subgroup [5].

Our arsenal of effective evidence-based therapies for $\mathrm{CM}$ is limited. Topiramate at daily doses of approximately $100 \mathrm{mg}$ is reported to be effective and reasonably well tolerated when used for the preventive treatment of $\mathrm{CM}$ (even in the presence of medication overuse) by two randomized, double-blind, placebo-controlled trials [13, 14]. In these trials $\mathrm{CM}$ is defined according to ICHD-2R classification, so that many patients included suffered from relatively mild forms of $\mathrm{CM}$ (with a number of migraine days between 15 and 20 per month). The mean number of monthly migraine days was reduced by $3.5 \pm 6.3$, compared with placebo $(-0.2 \pm 4.7)$ in one study [14] and by $5.6 \pm 6$ compared with placebo $(-4.1 \pm 6.1)$ in the other one [13]. These differences are statistically significant but they may be considered of little significance from a clinical point of view especially in a patient with a true daily headache.

A more recent option in the treatment of CM is onabotulinumtoxinA. Two double-blind, randomized, placebocontrolled clinical trials for the treatment of $\mathrm{CM}$ with onabotulinumtoxinA, the Phase III REsearch Evaluating Migraine Prophylaxis Therapy (PREEMPT) 1 and 2, as well as the pooled data from the two trials were recently published [12, 44, 45]. Pooled analyses demonstrate a mean decrease from baseline in frequency of headache days, with statistically significant between-group differences favouring onabotulinumtoxinA over placebo $(-8.4$ vs. $-6.6 ; P<0.001)$. CM is defined according to ICHD-
$2 \mathrm{R}$ classification and all doubts about study population features and clinical relevance of the results that we raised about use of topiramate in $\mathrm{CM}$ are valid for onabotulinumtoxinA too.

A hope for patients with $\mathrm{CM}$ might come from the neurostimulation techniques [15]. This option is very interesting but has to be evaluated by further studies and is not else available in the clinical practice.

Taking into account this scenery, we can evaluate the results obtained in chronic headache patients with longacting opioid therapies.

Saper et al. [18] assess a daily opioid treatment in selected patients with chronic intractable headache. From an initial cohort of 160 subjects, 70 (43.7 \%) are still using opioids daily at a 3-year follow-up evaluation. Forty-one (26\%) have an improvement of $50 \%$ on a pooled measure of frequency and duration. The authors conclude that opioid therapy may provide significant long-term relief to selected patients with chronic refractory headache, although in a minority of subjects. Thereafter, for $25 \%$ of the initially treated sample, results are excellent. An important finding is that many patients can be identified as either good or poor responders within the first 4 weeks. The principal concern is about dose violations that occurred at the rate of $16.5 \%$ per year despite meticulous efforts to minimize misuse.

While Saper et al. [18] use different opioids, Rothrock et al. $[20,21]$ prescribe only methadone to their subject population with daily intractable headache, according to a uniform and prospectively derived dosing schedule. They find that a significant minority of patients with refractory $\mathrm{CM}$ responds favourably to methadone (50\% or greater reduction in headache days per month) at a relatively low dose (ranging from 2.5 to $10 \mathrm{mg}$ TID), while patients who report no or minimal improvement at a low dose fail to experience a positive treatment response even if the dose is increased. While Saper et al. [18] report that many of those who initially appear to respond to chronic opioid therapy fail to maintain their initially favourable response, the patients treated by Rothrock [21] seem to maintain the benefit over time. The kind of opioid employed may do the difference. Methadone seems less likely to produce euphoria, tolerance and psychological addiction when compared with other opioids. Thereafter, unlike other opioids, methadone has the effect of antagonizing glutamate and may be less likely to produce or enhance central sensitization a phenomenon that may be a function of pro-nociceptive changes in NMDA receptors [46].

Given the refractory nature of their patients, Saper's and Rothrock's results suggest that in expert hands daily opioids provide an option for the treatment of some individuals with intractable headache. 
In conclusion, long-acting daily opioids may help a minority of patients with frequent and disabling headaches that fail to respond to other therapies. An individualized and careful balance of the risk and benefits is necessary and patients have to be cautiously monitored. Controlled trials about the use of long-acting opioids in refractory $\mathrm{CM}$ are advisable.

Conflict of interest The author certifies that there is no actual or potential conflict of interest in relation to this article.

\section{References}

1. Stovner LJ, Andree C (2010) Prevalence of headache in Europe: a review for the Eurolight project. J Headache Pain 11:289-299

2. Evers S, Afra J, Frese A, Goadsby PJ, Linde M, Sandor PS (2006) EFNS guideline on the drug treatment of migraine-report of an EFNS task force. Eur J Neurol 13:560-572

3. Evers S, Afra J, Frese A, Goadsby PJ, Linde M, May A, Sándor PS (2009) EFNS guideline on the drug treatment of migrainerevised report of an EFNS task force. Eur J Neurol 16(9):968-981

4. Silberstein SD (2000) Practice parameter: evidence-based guidelines for migraine headache (an evidence-based review): report of the Quality Standards Subcommittee of the American Academy of Neurology. Neurology 55(6):754-762

5. Manzoni GC, Bonavita V, Bussone G, Cortelli P, Narbone MC, Cevoli S, D'Amico D, De Simone R, Torelli P, on behalf of Associazione Neurologica Italiana Ricerca Cefalee (ANIRCEF) (2011) Chronic migraine classification: current knowledge and future perspectives. J Headache Pain 12(6):585-592

6. Headache Classification Subcommittee of the International Headache Society (2004) The international classification of headache disorders-2nd edn. Cephalalgia 24(1):1-160

7. Bigal ME, Serrano D, Buse D, Scher A, Stewart WF, Lipton RB (2008) Acute migraine medications and evolution from episodic to chronic migraine: a longitudinal population-based study. Headache 48:1157-1168

8. Bigal ME, Lipton RB (2009) Excessive opioid use and the development of chronic migraine. Pain 142:179-182

9. Buse DC, Pearlman SH, Reed ML, Serrano D, Ng-Mak D, Lipton RB (2012) Opioid use and dependence among persons with migraine: results of the AMPP Study. Headache 52:18-36

10. Vinson DR (2002) Treatment patterns of isolated benign headache in US emergency departments. Ann Emerg Med 39:215-222

11. Grazzi L, Andrasik F, D'Amico D, Usai S, Kass S, Bussone G (2004) Disability in chronic migraine patients with medication overuse: treatment effects at 1-year follow-up. Headache 44(7): 678-683

12. Diener HC, Dodick DW, Aurora SK, Turkel CC, De Gryse RE, Lipton RB, Silberstein SD, Brin MF, PREEMPT 2 Chronic Migraine Study Group (2010) Onabotulinumtoxin A for treatment of chronic migraine: results from the double-blind, randomized, placebo-controlled phase of the PREEMPT 2 trial. Cephalalgia 30(7):804-814

13. Silberstein S, Lipton R, Dodick D, Freitag FG, Ramadan ND, Mathew N, Brandes JL, Bigal M, Saper J, Ascher S, Jordan DM, Greenberg SJ, Hulihan J, on behalf of the Topiramate Chronic Migraine Study Group (2007) Efficacy and safety of topiramate for the treatment of chronic migraine: a randomized, doubleblind, placebo controlled trial. Headache 47:170-180

14. Diener H, Bussone G, Van Oene J, Lahaye M, Schwalen S, Goadsby PJ, on behalf of the TOPMAT-MIG-201(TOP-
CHROME) Study Group (2007) Topiramate reduces headache days in chronic migraine: a randomized, double-blind, placebocontrolled study. Cephalalgia 27:814-823

15. Proietti Cecchini A, Leone M, Manzoni GC, Torelli P, Bussone G (2012) Drug-resistant chronic migraine: the Italian GON project. Neurol Sci 33(1):S33-S35

16. Rothrock J, Parada V, Sims C, Key K, Walters N, Zweifler R (2005) Predictors of a negative response to topiramate therapy in patients with chronic migraine. Headache 45:932-935

17. Conway S, Delplanche C, Crowder J, Rothrock J (2005) Botox therapy for refractory chronic migraine. Headache 45:355-357

18. Saper J, Lake A, Hamel RL, Lutz TE, Branca B, Sims DB, Kroll MM (2004) Daily scheduled opioids for intractable head pain: long-term observations of a treatment program. Neurology 62: $1687-1694$

19. Robbins L (1999) Long-acting opioids for severe chronic daily headache. Headache Q 10:135-139

20. Rothrock JF (1999) Management of chronic daily headache utilizing a uniform treatment pathway. Headache 39:650-653

21. Rothrock JF (2008) Treatment-refractory migraine: the case for opioid therapy. Headache 48:850-854

22. Diamond S, Bigal ME, Silberstein S, Loder E, Reed M, Lipton RB (2007) Patterns of diagnosis and acute and preventive treatment for migraine in the United States: results from the American Migraine Prevalence and Prevention study. Headache 47:355-363

23. Vinson DR (2002) Treatment patterns of isolated benign headache in US emergency departments. Ann Emerg Med 39:215-222

24. Colman I, Rothney A, Wright SC, Zilkalns B, Rowe BH (2004) Use of narcotic analgesics in the emergency department treatment of migraine headache. Neurology 62:1695-1700

25. Valade D, Lucas C, Calvel L, Plaisance P, Derouet N, Meric G, Lanteri-Minet M, Giroud M (2011) Migraine diagnosis and management in general emergency departments in France. Cephalalgia 31(4):471-480

26. Akpunonu BE, Mutgi AB, Federman DJ, Volinsky FG, Brickman K, Davis RL, Gilbert C, Asgharnejad M (1995) Subcutaneous sumatriptan for treatment of acute migraine in patients admitted to the emergency department: a multicenter study. Ann Emerg Med 25:464-469

27. Martelletti P, Farinelli I, Steiner TJ, on behalf of the Working Group for Specialist Education, WHO's Global Campaign to Reduce the Burden of Headache Worldwide (Lifting The Burden) (2008) Acute migraine in the emergency department: extending European principles of management. Intern Emerg Med 3(1): S17-S24

28. Friedman BW, Kapoor A, Friedman MS, Hochberg ML, Rowe BH (2008) The relative efficacy of meperidine for the treatment of acute migraine: a meta-analysis of randomized controlled trials. Ann Emerg Med 52:705-713

29. Sumamo Schellenberg E, Dryden DM, Pasichnyk D, Ha C, Vandermeer B, Friedman BW, Colman I, Rowe BH (2012) Acute migraine treatment in emergency settings. Comparative effectiveness review No. 84. (Prepared by the University of Alberta Evidence based Practice Center under Contract No. 290-200710021-I.). AHRQ Publication No. 12(13)-EHC142-EF. Agency for Healthcare Research and Quality: Rockville, MD. www. effectivehealthcare.gov/reports/final.cfm

30. Alemdar M, Pekdemir M, Selekler HM (2007) Single-dose intravenous tramadol for acute migraine pain in adults: a singleblind, prospective, randomized, placebo-controlled clinical trial. Clin Ther 29:1441-1447

31. Engindeniz Z, Demircan C, Karli N (2005) Intramuscular tramadol vs. diclofenac sodium for the treatment of acute migraine attacks in emergency department: a prospective, randomized, doubleblind study. J Headache Pain 6:143-148 
32. Bigal ME, Lipton RB (2009) Excessive opioid use and the development of chronic migraine. Pain 142:179-182

33. Bigal ME, Serrano D, Buse D, Scher A, Stewart WF, Lipton RB (2008) Acute migraine medications and evolution from episodic to chronic migraine: a longitudinal population-based study. Headache 48:1157-1168

34. Bahra A, Walsh M, Menon S (2003) Does chronic daily headache arise de novo in association with regular use of analgesics? Headache 43:179-190

35. Limmroth V, Katsarava Z, Fritsche G (2002) Features of medication overuse headache following overuse of different acute headache drugs. Neurology 59:1011-1014

36. Diener HC (2011) Detoxification for medication overuse headache is not necessary. Cephalalgia 32(5):423-427

37. Kim JH, Suh SI, Seol HY (2008) Regional grey matter changes in patients with migraine: a voxel-based morphometry study. Cephalalgia 28:598-604

38. Welch KMA, Nagesh V, Aurora SK, Gelman N (2001) Periaqueductal gray matter dysfunction in migraine: cause or the burden of illness? Headache 41:629-637

39. Mathew NT ((2011) Pathophysiology of chronic migraine and mode of action of preventive medications. Headache 51(S2): 84-92

40. Johnson JL, Hutchinson MR, Williams DB, Rolan P (2012) Medication-overuse headache and opioid-induced hyperalgesia: a review of mechanisms, a neuroimmune hypothesis and a novel approach to treatment. Cephalalgia 33(1):52-64

41. Rizzoli P, Loder EW (2011) Tolerance to the beneficial effects of prophylactic migraine drugs: a systematic review of causes and mechanisms. Headache 51:1323-1335

42. Langemark M, Olesen J (1984) Drug abuse in migraine patients. Pain 19:81-86

43. Tennant FS Jr, Rawson RA (1982) Outpatient treatment of prescription opioid dependence: comparison of two methods. Arch Intern Med 142:1845-1847

44. Aurora SK, Dodick DW, Turkel CC, DeGryse RE, Silberstein SD, Lipton RB, Diener HC, Brin MF, on behalf of PREEMPT 1 Chronic Migraine Study Group (2010) OnabotulinumtoxinA for treatment of chronic migraine: results from the double-blind, randomized, placebo-controlled phase of the PREEMPT 1 trial. Cephalalgia 30:793-803

45. Dodick DW, Turkel CC, DeGryse RE, Aurora SK, Silberstein SD, Lipton RB, Diener HC, Brin MF, on behalf of the PREEMPT Chronic Migraine Study Group (2010) Onabotulinumtoxin A for treatment of chronic migraine: pooled results from the doubleblind, randomized, placebo-controlled phases of the PREEMPT clinical program. Headache 50:921-936

46. Tompkins DA, Campbell CM (2011) Opioid-induced hyperalgesia: clinically relevant or extraneous research phenomenon? Curr Pain Headache Rep 15:129-136 